\title{
Anastomosis tubo-tubaria en el Instituto Materno Infantil de Bogotá. Diez años de experiencia
}

\author{
Carlos Pacheco Consuegra*; José W. León**
}

\begin{abstract}
RESUMEN: Se revisan 90 historias clínicas de pacientes que consultan a la Clínica de Infertilidad del Instituto Materno Infantil de Bogotá para recanalización tubárica posterior a esterilización quirúrgica en el período del 1o. de enero de 1983 al 31 de diciembre de 1992. Se practicó la evaluación completa de la pareja para determinar la presencia de factores de infertilidad diferentes a la ligadura tubárica. El factor tuboperitoneal fue evaluado por laparoscopia previo en la gran mayoría de los casos pero se omitió en 18 casos basados en los datos de la cirugía. Se realizó la anastomosis con técnica microquirúrgica, lupas con aumento de $4 X$ para magnificación y suturas Vicryl 7-0 u 8-0, irrigación con Lactato Ringer con heparina y corticoides y antibióticos. Se realizaron 53 anastomosis, 41 posteriores a bandas de silastic y 12 a ligadura por técnica de Pomeroy, con una tasa global de embarazo a término del $62 \%$, embarazo ectópico del $4 \%$ y aborto en $2 \%$. El resultado fue mejor para las bandas que para el Pomeroy (66\% vs. $50 \%)$. No se encontró diferencia para el tiempo de ligadura ni segmento anastomosado. El resultado de la anastomosis intramural itsmica $(75 \%)$ demuestra la utilidad de las lupas aún en este segmento. La tasa de embarazo para longitud tubárica residual mayor de $6 \mathrm{cms}$. (72\%) fue mejor que para longitudes de $4-6 \mathrm{cms}$. (55\%). Se demuestra la utilidad de las lupas en microcirugía, la longitud tubárica residual como factor pronóstico y las tasas y tiempos de embarazo.
\end{abstract}

PALABRAS CLAVES: Esterilización quirúrgica, Anastomosis tubárica, Infertilidad.

SUMMARY : 90 charts of patients at the Infertility Clinic at the Instituto Materno Infantil were reviewed. The patient underwent tubal reanastomosis after surgical sterilization from january 1983 to december 1992 . Complete evaluation of the couples was done to determine other causes of infertility different from the tubal ligation. Laparoscopy was done in all the cases except 18, were the data was collected from the surgical records. Microquirurgic technic reanastomosis was done with $4 \mathrm{X}$ magnifing lenses, and Vicryl 7.0 or 8.0 sutures, Ringer lactate irrigation with heparin and corticosteroids and antibiotics. 53 anastomosis were performed, 41 after silastic bands and 12 after ligation with Pomeroy technic. The global pregnancy at term was $62 \%$, ectopic pregnancy $4 \%$ and abortion $2 \%$. The results were better for the patients with ligation with bands than Pomeroy $(66 \% \mathrm{vs} .50 \%)$. Thee was no difference for the length in time of the ligation or the segments of the anastomosis. The results with of the intramural itsmic anastomosis $(\mathbf{7 5 \%})$ demonstrates the utility of the magnifing glases even in this portion of the tube. The pregnancy rate for a longer tube segment greater than $6 \mathrm{cms}$. (72\%) was better than for $4-6 \mathrm{cms}$. (55\%). The utility of the magnifing glases in tubal anastomosis, the prognosis based on the tubal length and the pregnancy rates are demonstrated.

KEY WORDS: Surgical sterilization, anastomosis tubal, infertility.

\section{Introducción}

Con la creciente difusión de la esterilización quirúrgica como método de control de la natalidad, se presenta en forma concurrente una demanda de cirugía reconstructiva de trompas. Las causas mas frecuentes para esta solicitud son el cambio de pareja, pérdida de un hijo y ocasionalmente el deseo de un nuevo hijo con la misma pareja (1).

Inicialmente se utilizaron en los procedimientos reconstructivos técnicas quirúrgicas convencionales y posteriormente técnicas microquirúrgicas, encontrándose en general una evidencia de la superioridad de éstas últimas (2-3).

Aunque desde varios años antes se habían practicado microcirugías en el I.M.I., ésta se inició en forma protocolizada y más regular a partir de Julio de 1983 en

Profesor Asociado.

Residente III.

Departamento de Obstetricia y Ginecología.

Universidad Nacional de Colombia.

Instituto Materno Infantil - Santafé de Bogotá. la medida que se fueron resolviendo algunos obstáculos. Los resultados de la aplicación de técnicas microquirúrgicas para la anastomosis tubárica durante estos 10 años se presentan en éste artículo.

\section{Materiales y métodos}

Se incluyeron en el estudio todas las pacientes que ingresaron a la Clínica de Infertilidad del Instituto Materno Infantil de Bogotá en el período comprendido desde el $1^{\circ}$ de enero de 1983 hasta el 31 de diciembre de 1992. El procedimiento de ingreso incluyó una entrevista de información con la pareja, la elaboración de una historia clínica, se practicó el examen físico y se realizaron los exámenes encaminados al estudio de los factores masculino, ovárico, tuboperitoneal, cervical e inmunológico cuando el caso lo ameritaba.

Para el estudio del factor tuboperitoneal se realizó una laparoscopia diagnóstica aunque en algunos casos se omitió la laparoscopia basados en el informe de la cirugía previa, sobre todo en casos de ligadura por banda de silastic realizada en Profamilia de Bogotá. 
Se utilizó técnica microquirúrgica con lupas de $4 X$ para magnificación, suturas finas del tipo Vicryl 7-0 y 80 , instrumental de microcirugía, irrigación permanente con solución de Lactato Ringer heparinizado (5000 UI por cada $1000 \mathrm{ml}$. de solución), en la gran mayoría se agregaron corticoides del tipo de la hidrocortisona o dexametasona, antibióticos profilácticos y en un porcentaje apreciable antihistamínicos intra y postoperatorios parenterales.

Además de la descripción quirúrgica usual, el registro de la información para este estudio se realizó en una historia precodificada que además de los datos de identificación, antecedentes y diagnóstico, incluía la longitud final de los muñones, segmentos anastomosados, intervalo cirugía-embarazo, localización del embarazo y tiempo de seguimiento hasta la elaboración de este informe. Los datos se analizaron en computador utilizando el programa de investigación epidemiológica EPI-INFO Versión 5.01.

\section{Resultados}

En el período de estudio ingresaron a la Clínica de Infertilidad un total de 855 parejas, de las cuales 90 pacientes consultaron para reanastomosis tubárica después de ligadura de trompas con banda de silastic en 57 casos (63\%), Pomeroy en $32(36 \%)$ y fimbriectomía en $1(1 \%)$.

La motivación para la reversión de la ligadura fue: un nuevo compañero en 59 casos (66\%), deseo de un nuevo hijo en la misma unión en 14 casos (15\%), muerte de un hijo en $9(10 \%)$ y 8 casos sin dato (9\%). El tiempo promedio de ligadura fue de 74.8 meses (DS de 39.7, rango 10-180 meses) y la edad promedio de las pacientes fue de 33 años (DS de 3.4, rango 25-40).

Un total de 36 casos no se operaron: 27 deserciones, 2 embarazos durante el estudio con ligadura por Pomeroy, 2 sin datos y 5 pacientes dadas de alta por mal pronóstico. Se dieron de alta 3 casos de Pomeroy, 1 fimbriectomía y 1 de bandas (por fibrosis de los segmentos).

Se practicó laparoscopia diagnóstica a 52 pacientes; fueron dados de alta por mal pronóstico 3 de los 16 con Pomeroy (19\%) y 1 de 35 con banda de silastic (3\%) y 1 fimbriectomía. El 32\% (13/41) de las bandas de silastic y $42 \%(5 / 12)$ de los Pomeroy se operaron sin laparoscopia, basados en el informe del tipo de cirugía.

Se realizaron 54 cirugías (53 anastomosis y 1 implantación tubo-uterina unilateral). Los resultados de las anastomosis se describen en la Tabla 1, incluyendo todos los casos (un caso extraviado desde el momento de la cirugía y los casos con longitudes residuales menores de $4 \mathrm{cms}$ ). No se incluyó la paciente con implantación tubouterina (quien no se embarazó).

Se comparó el resultado de la cirugía (embarazos) con la duración de la esterilización en años y no se encontró diferencia significativa entre los grupos hasta los 10 años de ligadura para cada técnica ni en el grupo total; para duraciones mayores de 10 años no fue posible realizar un análisis por el tamaño reducido de la muestra.

Se analizaron los resultados de acuerdo con los segmentos anastomosados y embarazo (Tabla 2): itsmico-itsmico (IS-IS), itsmico-ampular (IS-AM), intramural-itsmico (IMIS) y ampular-ampular (AM-AM). Aquellos casos con la misma técnica bilateral o en el único anexo presente, permiten observaciones en este sentido. En la cirugía bilateral por bandas (IS-IS/IS-IS e IM-IS/IM-IS) se obtuvo embarazo viable en $23 / 34(68 \%)$ y $2 / 2(100 \%)$ respectivamente y en los casos de Pomeroy (IS-IS/IS-IS) en $2 /$ $4(50 \%)$. En la cirugía de anexo único (IS-IS, IS-AM, AM-AM, IM-IS) el resultado fue $2 / 3(67 \%)$ para las bandas y $2 / 4(50 \%)$ para el Pomeroy. En la anastomosis IM-IS uni o bilateral se lograron 3/4 (75\%) embarazos a término confirmando la utilidad de las lupas aún en este segmento.

Tabla 2

ANASTOMOSIS Y SEGMENTO TUBARICO

\begin{tabular}{|l|cc|cc|}
\hline Segmento & \multicolumn{2}{|c|}{$\begin{array}{c}\text { Banda } \\
\text { No. Embarazos }\end{array}$} & $\begin{array}{c}\text { Pomeroy } \\
\text { No. embarazos }\end{array}$ \\
\hline IS-IS/IS-IS & 34 & $26^{*}$ & 4 & 2 \\
IS-AM/IS-IS & & 2 & 4 & 2 \\
IM-IS/IM-IS & 2 & 2 & & \\
IM-IS/IS-IS & 1 & 0 & & \\
AM-AM/IS-AM & 1 & 0 & 1 & 0 \\
IS-IS & 2 & 1 & 2 & 1 \\
IS-AM & & & 1 & 1 \\
AM-AM & 1 & 1 & 12 & 6 \\
IM-IS & 41 & $30^{*}$ & 12 \\
Total & \multicolumn{2}{|l}{} \\
\hline
\end{tabular}

* Incluye 2 ectópicos y un aborto

IS: Itsmico; AM: ampular; IM: intramural

Tabla 1

RESULTADO DE LA ANASTOMOSIS

\begin{tabular}{|lc|cc|cc|cc|}
\hline Técnica & Total & Aborto No. \% & Ectópico No. $\%$ & Emb. Viable No. & $\%$ \\
\hline Banda & 41 & 1 & 2 & 2 & 5 & 27 & 66 \\
Bilateral & 38 & 1 & & 2 & & 25 & 66 \\
Unilateral & 13 & & & & & 2 & 67 \\
Pomeroy & 12 & & & & & 6 & 50 \\
Bilateral & 6 & & & & & 2 & 33 \\
Unilateral & 6 & & 2 & 2 & 4 & 33 & 62 \\
Total & 53 & 1 & 2 & & & \\
\hline
\end{tabular}


Para investigar la relación entre la longitud tubárica residual y el resultado final (en términos de tasa de embarazos) se clasificó arbitrariamente la longitud partiendo de longitudes inferiores a $4 \mathrm{cms}$. (longitud mínima requerida en la literatura para lograr buenos resultados) y por el anexo de mayor longitud (Tabla 3 ).

Tabla 3

ANASTOMOSIS Y LONGITUD TUBARICA FINAL

\begin{tabular}{|c|c|c|c|c|c|c|c|c|c|c|}
\hline $\begin{array}{l}\text { Longitud } \\
\text { (Cms) }\end{array}$ & No & Bar & & EV & $\begin{array}{l}\text { Pon } \\
\text { No }\end{array}$ & $\begin{array}{l}\text { neroy } \\
\text { EV }\end{array}$ & No & To & & $\mathrm{EV}$ \\
\hline$<4$ & 2 & & 1 & & & & 2 & & 1 & \\
\hline $4.0-6$ & 16 & 1 & & 11 & 6 & 2 & 22 & 1 & & 13 \\
\hline $6.1-8$ & 19 & & 1 & 14 & 4 & 2 & 23 & & & 16 \\
\hline $8.1-10$ & 3 & & & 2 & 1 & 1 & 4 & & & 3 \\
\hline Sin dato & 1 & & & & 1 & 1 & 2 & & & 1 \\
\hline Total & 41 & 1 & 2 & 27 & 12 & 6 & 53 & 1 & & 33 \\
\hline$(\%)$ & & 2 & 5 & 66 & & 50 & & 2 & & 62 \\
\hline
\end{tabular}

Ab: aborto; Ec: ectópico; EV: embarazo viable.

Todas las pacientes que se operaron sin laparoscopia presentaron una longitud final adecuada con excepción de una ligadura con banda que mostró gran fibrosis del segmento proximal bilateralmente y requirió la implantación tubouterina descrita antes. En contraste con esto, las estudiadas con laparoscopia en 3 casos de bandas tenían doble ligadura ( 2 terminaron con longitudes de 3.5 y $3.0 \mathrm{cms}$. y en estos casos ocurrió solamente un embarazo ectópico).

Del análisis de la Tabla 3 se observa que no hay embarazo intraútero por debajo de $4 \mathrm{cms}$ de longitud residual. La tasa de embarazo viable fue mejor para las bandas de silastic que para el Pomeroy ( $66 \%$ vs $50 \%$ ), con una tasa total de $62 \%$ viables, $2 \%$ de abortos y de $4 \%$ para el embarazo ectópico.

La tasa acumulativa global de embarazos muestra que a los 7 meses postcirugía se lograron la mitad de los embarazos, mientras que para las bandas de silastic la mitad de los embarazos se lograron a los 5 meses; la totalidad de los embarazos se logró en 12 meses para el Pomeroy y 26 meses para la banda de silastic (Tabla 4).

Inicialmente se recomendó a las pacientes la anticoncepción por tres meses post-cirugía, pero luego de observar varios embarazos en los dos primeros meses, sin complicaciones, se les ha aconsejado evitar el embarazo solamente en el ciclo de cirugía.

\section{Discusión}

Con el incremento de la esterilización quirúrgica como método de control natal ha crecido también la demanda por la reversión del procedimiento y como consecuencia el Instituto Materno Infantil de Bogotá registra un incremento gradual en las consultas por este
Tabla 4

ANASTOMOSIS Y TASA ACUMULATIVA DE EMBARAZOS

\begin{tabular}{|lllll|}
\hline \multirow{2}{*}{ Meses } & \multirow{2}{*}{ No. } & \multicolumn{3}{c|}{ Tasa $(\%)$} \\
& Embarazo & Total & Banda & Pomeroy \\
\hline 1 & 4 & 7.5 & 9.8 & \\
2 & 10 & 26.4 & 29.3 & 16.7 \\
3 & 3 & 32.0 & 36.6 & \\
4 & 6 & 43.4 & 48.8 & 25.0 \\
5 & 2 & 47.2 & 51.2 & 33.3 \\
6 & 1 & 49.1 & 53.7 & \\
7 & 1 & 50.9 & 56.1 & \\
8 & 2 & 54.7 & 61.0 & \\
11 & 1 & 56.6 & & 41.7 \\
12 & 2 & 60.4 & 63.4 & 50.0 \\
15 & 1 & 62.3 & 65.9 & \\
16 & 1 & 64.2 & 68.3 & \\
22 & 1 & 66.0 & 70.7 & \\
26 & 1 & 67.9 & 73.2 & \\
\hline
\end{tabular}

motivo. Gomel (1) ha informado como motivos de solicitud de reanastomosis, el cambio del estado marital en el $63 \%$, con el mismo compañero $10 \%$ y muerte de un hijo en $17 \%$, tragedia en $4 \%$ y razones psicológicas en $6 \%$. Por otro lado Spivak (4) informa como causas el $66 \%$ por cambio de estado, $30 \%$ con igual compañero y $4 \%$ por muerte de un hijo. En nuestro estudio las tres primeras causas fueron el $66 \%, 15 \%$ y $10 \%$ respectivamente.

El resultado global de la anastomosis postligadura en términos de embarazos a término $(62 \%)$, aborto $(2 \%)$ y ectópico (4\%) es comparable al informado en la literatura internacional como se ilustra en la Tabla 5.

Los factores pronósticos para la anastomosis postesterilización que se han mencionado en la literatura y que la American Fertility Society (AFS) incluye en su clasificación de obstrucción secundaria a ligadura tubárica (14) son: edad de la paciente, tipo de esterilización, intervalo de esterilización, longitud residual y tipo de segmento anastomosado.

La edad tiene un efecto negativo reconocido sobre la fertilidad y en relación con la cirugía tubárica pueden existir además intervalos más largos de esterilización, alteraciones de la ovulación, etc. Silber (15) no encontró diferencia en la tasa de embarazo ni el tiempo de latencia para un grupo entre 21 y 36 años, pero TeVelde (13) si reporta la influencia de la edad en un grupo de pacientes mayores de 40 años. En nuestra experiencia, el embarazo se presentó uniformemente en todas las edades entre 25 y 40 años.

Los resultados de acuerdo al tipo de esterilización muestran tasas variables en los informes de la literatura con mejores resultados para la banda de silastic, clip de 
Tabla 5

ANASTOMOSIS TUBO-TUBARIA

\begin{tabular}{|c|c|c|c|c|c|c|}
\hline Autor & Año & No. & Intraut. & Término & Aborto & Ectópico \\
\hline Siegler \& Kontopoulos (5) & 1979 & 16 & $8(50)$ & & & $1(6)$ \\
\hline Frantzen \& Schossler (6) & 1982 & 28 & $12(43)$ & $12(43)$ & $2(7)$ & \\
\hline Fayez \& Suliman (7) & 1982 & 31 & $18(58)$ & $15(48)$ & $2(7)$ & $1(3)$ \\
\hline DeCherney et al. (8) & 1983 & 124 & $84(68)$ & $72(58)$ & $8(6)$ & \\
\hline Spivak et al. (4) & 1986 & 89 & $49(55)$ & $40(45)$ & $9(10)$ & $6(5)$ \\
\hline Rock et al. (9) & 1987 & 80 & & $49(61)$ & & \\
\hline Pérez \& Abdala (Col.) (10) & 1989 & 44 & $31(66)$ & $25(57)$ & $4(9)$ & $2(5)$ \\
\hline Gómez \& Estela (Col.) (11)* & 1990 & 25 & & & $14(56)$ & \\
\hline Trimbos-Kemper $(12)^{* *}$ & 1990 & 78 & $35(45)$ & $26(33)$ & $9(12)$ & $3(4)$ \\
\hline Te Velde et al. (13) & 1990 & 215 & $156(73)$ & $132(61)$ & $19(9)$ & $5(2)$ \\
\hline Presente estudio-I.M.I. & 1995 & 53 & $34(64)$ & $33(62)$ & $1(2)$ & $2(4)$ \\
\hline
\end{tabular}

* Sólo incluye pacientes con seguimiento.

**Pacientes mayores de 40 años.

Hulka y ligadura simple y mas bajos para la coagulación, excepto Seiler (16) quien además informa de intervalos más largos y menor posibilidad quirúrgica (Tabla 6).

Vásquez (18) describió aplanamiento y pólipos en la mucosa del muñón proximal post-esterilización con deciliación por parches visibles al microscopio electrónico, hallazgo muy común en intervalos mayores de 5 años, poco común entre 3 y 4 años y ausente en menores de 3 años. Sin embargo muchos informes no encuentran una relación entre este intervalo y la tasa de embarazo (4, 1516). En este estudio no se encontró diferencia en los resultados entre uno y diez años de intervalo.

Se ha dicho (15) que «el factor con mayor influencia en el éxito es la longitud tubárica total, en presencia de fimbrias y un centímetro de la porción ampular intacto». Silber (15) informa embarazos a término en $100 \%$ de casos con más de $5 \mathrm{cms}$., $82 \%$ entre 4 y $5,57 \%$ entre 3 y 4 y $18 \%$ en menos de 3 cms. Spivak (4) $73 \%$ en más de 6 cms., $42 \%$ entre 4 y 6 y $20 \%$ con menos de $4 \mathrm{cms}$. En nuestra experiencia, con longitudes menores de $4 \mathrm{cms}$. sólo obtuvimos un embarazo ectópico aunque el número de casos fue muy bajo, $55 \%$ de embarazos a término entre
4 y $6 \mathrm{cms}$. y $72 \%$ con más de $6 \mathrm{cms}$. También se ha descrito una relación inversa entre la longitud final de la trompa y el tiempo de embarazo $(1,15)$ con mejores resultados para longitudes mayores de $6 \mathrm{cms}$. pero otros autores no encuentran dicha relación (9); esto no fue posible analizarlo en el presente trabajo por el bajo número de casos menores de $4 \mathrm{cms}$.

Finalmente no encontramos diferencia en los resultados analizados según los segmentos anastomosados, ni tampoco cuando la anastomosis fue uni o bilateral, coincidiendo con otros autores $(1,9,16)$ en que esto no parece ser determinante del éxito. Rock (19) no encontró diferencia en la tasa de embarazos con la utilización de lupas o microscopio en la recanalización diferente a la intramural itsmica y en nuestra experiencia obtuvimos resultados excelentes en la anastomosis de estos segmentos, reafirmando la utilidad de las lupas aún en este tipo de cirugía.

En resumen, la experiencia en el Instituto Materno Infantil muestra un incremento en la demanda para reanastomosis tubárica postligadura por causas similares a las descritas en la literatura; es posible evitar la laparoscopia en pacientes candidatos a reanastomosis

Tabla 6

TASAS (\%) DE EMBARAZO SEGUN TIPO DE ESTERILIZACION

\begin{tabular}{|lccccc|}
\hline Autor & Año & Clip & Banda & Ligadura & Coagulación Irving \\
\hline Seiler (16) & 1983 & 50 & 63 & 68 & 75 \\
Siegler et al. (17) & 1985 & 84 & 72 & 50 & 41 \\
Spivak et al. (4) & 1986 & & 80 & 52 & 61 \\
Rock et al. (9) & 1987 & 86 & & 52 & 33 \\
Pérez \& Abdala (10) & 1989 & 71 & & 45 & 33 \\
Trimbos-Kemper (12) & 1990 & $63^{*}$ & & 56 & 49 \\
te Velde et al. (13) & 1990 & $67^{*}$ & & 75 & \\
Presente estudio-I.M.I. & 1995 & 66 & & 50 & \\
\hline
\end{tabular}

* Clip y banda. 
basados en los datos de la cirugía previa y eventualmente en las ligaduras por bandas de silastic. Los resultados con el manejo de lupas son comparables a otros estudios manejados con microscopio aún en la anastomosis itsmicointramural; no existieron diferencias en los resultados cuando se analizaron con relación a la edad por debajo de los 40 años, al intervalo de esterilización, segmento anastomosado ni a la uni o bilateralidad del procedimien- to. Los resultados fueron mejores para la ligadura con banda de silastic que para la técnica de Pomeroy y mejores con longitudes tubáricas residuales superiores a $6 \mathrm{cms}$.

\section{Agradecimientos}

- A Natividad Rodríguez, Auxiliar de Enfermería por su labor en el manejo del archivo de historias y la localización y seguimiento de pacientes.

\section{BIBLIOGRAFIA}

1. Gomel V: Microsurgical reversal of female sterilization: A reappraisal. Fertil Steril, 1980; 33: 587-597.

2. Bateman BG., Nunley Jr. WC., Kitchin III JD. Surgical management of distal tubal obstruction - are we making progress? Fertil Steril, 1987; 48: 523.

3. Fayez JA, Suliman SO. Infertility surgery of the oviduct; comparison between macrosurgery and microsurgery. Fertil Steril, 1982; 37: 73.

4. Spivak MM., Librach CL., Rosenthal DM. Microsurgical reversal of sterilization: A six-year study. Am. J. Obstet. 1986; 154: 355-361.

5. Siegler AM., Kontopoulos V. An analysis of macrosurgical techniques in the management of the tuboperitoneal factor in infertility. Fertil Steril, $1979 ; 32: 377$.

6. Frantzen C., Schlosser HW. Microsurgery and postinfections tubal infertility. Fertil Steril, 1982; 38: 397.

7. Fayez JA., Suliman SO. Infertility surgery of the oviduct: Comparison between macrosurgery and microsurgery. Fertil Steril, 1982;37: 73.

8. DeCherney HH., Mezer HC., Naftolin F. Analysis of failure of microsurgical anastomosis after midsegment, non-coagulation tubal ligation. Fertil Steril, 1983; 39: 618.

9. Rock JA., Guzick DS., Katz E., Zacur HA., King TM. Tubal anastomosis: pregnancy success following reversal of Falope ring or monopolar cautery sterilization. Fertil Steril, 1987; 48: 13.

10. Pérez LE., Abdala LT. Microcirugía ginecológica en infertilidad: 7 años de experiencia. Revista Colombiana de Obstetricia y Ginecología, 1989; XL (2):137.
11. Gómez G, Estela S. Reanastomosis tubárica y otras técnicas microquirúrgicas en infertilidad. Revista Colombiana de Obstetricia y Ginecología, 1990; XLI (3): 163.

12. Trimbos-Kemper TCM. Reversal of sterilization in women over 40 years of age: a multicenter survey in the Netherlands. Fertil Steril, 1990; 53: 575.

13. Te Velde Er, Boer Me, Looman CWN, Habbema JDF. Factors influencing success or failure after reversal of sterilization: a multivariate approach. Fertil Steril, 1990; 54: 270.

14. The American Fertility Society. The American Fertility Society classifications of adnexal adhesions, distal tubal occlusion, tubal occlusion secondary to tubal ligation, tubal pregnancies, Mullerian anomalies and intrauterine adhesions. Fertil Steril, 1988; 49: 944.

15. Silber SJ, Cohen R: Microsurgical reversal of tubal sterilization: Factors affecting pregnancy rate, with long-term follow-up. Obstet. Gynecol. 1984; 64: 679.

16. Seiler JC. Factors influencing the outcome of microsurgical tubal ligation reversals. Am. J. Obstet. Gynecol. 1983; 146: 292.

17. Siegler AM., Hulka J., Peretz A. Reversibility of female sterilization. Fertil Steril, 1985; 43: 499.

18. Vásquez G., Winston RML., Boeckx W., Brosens I. Tubal lesions subsequent to sterilization and their relation to fertility after attempts at reversal. Am. J. Obstet. Gynecol. 1980.

19. Rock A., Bergquist CA., Kimball AW., Zacur HA., King TM. Comparison of the operating microscope and loupe for microsurgical tubal anastomosis: a randomized clinical trial. Fertil Steril, 1984; 41: 229. 